\section{Retroperitoneal hand-assisted laparoscopic nephrectomy and partial nephrectomy}

\section{Nosratollah Nezakatzgoo, Janet L. Colli, Matthew Mutter, Sheg Aranmolate, Robert Wake}

Department of Urology, University of Tennessee Health Science Center, Memphis, TN, USA

\section{Abstract}

The purpose of the present paper is to describe our technique and experience with retroperitoneal hand-assisted laparoscopic (HAL) nephrectomies as an alternative to the transperitoneal approach. Eight retroperitoneal HAL nephrectomies and one partial nephrectomy were performed. Several excisional techniques were employed incorporating the Harmonic scalpel or Ligasure device. Hemostatic agents were used to cover the renal defect. Surgical bolsters were sutured to the renal capsule with pleget reinforcements to aid in hemostasis. The average operative time was $210 \mathrm{~min}$ and estimated blood loss 110 $\mathrm{mL}$. Mean change in hematocrit was 3 units and creatinine was 1 point. No patient required a transfusion. There were no major complications, with a mean follow-up of fourteen months. On average, patients resumed oral intake in 2 days, and were discharged in 3 days. Pathological examination revealed that two lesions were benign and seven malignant. Tumor diameter averaged $3 \mathrm{~cm}$. There were no positive surgical margins. In conclusion, we have demonstrated the feasibility of retroperitoneal laparoscopic hand-assisted nephrectomy and partial nephrectomy surgery for solid renal masses.

\section{Introduction}

The initial technique for open and partial nephrectomy, as described by Licht and Novick in 1980, included a retroperitoneal approach using a flank incision. ${ }^{1}$ Subsequently, over the years less invasive approaches to nephrectomy and partial nephrectomy have been developed including: transperitoneal hand-assisted laparoscopy (HAL), laparoscopic and robotic assisted. ${ }^{2,3}$ The concept of hand-assisted laparoscopy was introduced in 1996 when Bannenberg and colleagues performed the first HAL nephrectomy in a pig. ${ }^{4}$ One year later, Nakada et al. performed the first human handassisted laparoscopic nephrectomy in a patient with a chronically infected kidney. ${ }^{5}$ Since this initial case report, many investigators have reported their experiences performing complex hand-assisted laparoscopic urologic procedures, including radical nephrectomies, ${ }^{6}$ nephroureterectomies, ${ }^{7,8}$ and donor nephrectomies. ${ }^{9}$

Current hand-assisted laparoscopy utilizes all the principles of standard transperitoneal laparoscopy and it offers the surgeon direct hand contact with the operative field, maximizing tactile feedback and thereby facilitating superior dissection, hemostatic control and suturing. ${ }^{6}$ In essence, HAL combines the advantages of laparoscopy with quicker and safer organ retrieval offered by open surgery with fewer complications., ${ }^{2,3}$ Indications for HAL include radical, donor, and partial nephrectomies, and as aforementioned, hand assisted laparoscopy is traditionally performed through transperitoneal methods. Overall, transperitoneal approaches are safe in experienced hands; however, in certain patient populations it is advantageous to remain extra-peritoneal. This idea is of particular significance in patients with advanced cirrhosis who are posed with an increased risk of complications if the peritoneal cavity is breeched or entered. In addition, this retroperitoneal technique is advantageous in patients who have undergone previous abdominal surgery, and may have excessive peritoneal adhesions. Furthermore, this approach is beneficial in patients whom are on peritoneal dialysis (PD), because the peritoneal cavity is not violated and PD does not have to be stopped postoperatively. Therefore, to avoid such potential intraperitoneal complications, (especially in patients with intra-abdominal co-morbidites), we present a technique for retroperitoneal handassisted laparoscopic nephrectomy and partial nephrectomy. Retroperitoneal hand-assisted nephrectomy has been described for donor nephrectomies, ${ }^{10-15}$ but has not previously been depicted for nephrectomies performed for other reasons such as renal mass/cancer. In this article, we describe techniques and review outcomes of retroperitoneal HAL nephrectomy (performed for solid renal mass) in comparative experience with standard transperitoneal techniques.

\section{Materials and Methods}

The patient is placed in the semilateral decubitus position using slight table flexion. An arm board is utilized to support the anterior arm and 3-inch cloth tape secures the patient to the table. This allows the patient to be rotated both away and toward the surgeon as necessary.

The port configuration for both left- and
Correspondence: Janet Colli, Department of Urology, University of Tennessee Health Science Center, 910 Madison Avenue, Room 409, 38163 Memphis, TN, USA.

Tel. +1.901.448.1026 - Fax: +1.901.448.1122.

E-mail: jcolli.tulane@gmail.com;

jcolli@uthsc.edu

Key words: laparoscopic, nephrectomy, surgical procedure.

Contributions: each author to this study participated in the: i) conception and study design or analysis and interpretation of data; ii) drafting the article or revising it critically; and iii) final approval of the version to be published.

Conflict of interests: the authors declare no potential conflict of interests.

Received for publication: 22 May 2013.

Revision received: 5 September 2013.

Accepted for publication: 12 September 2013.

This work is licensed under a Creative Commons Attribution NonCommercial 3.0 License (CC BYNC 3.0).

(C) Copyright N. Nezakatzgoo et al., 2013

Licensee PAGEPress, Italy

Surgical Techniques Development 2013; 3:e3 doi:10.4081/std.2013.e3

right-sided nephrectomy (right-handed surgeon) is illustrated in Figures 1 and 2. For a left-handed surgeon, a mirror image of the right-sided configuration is used and viceversa for a left-sided configuration. The surgery generally is performed with three ports: a hand port, camera port and working port. This configuration allows the hand to access the renal hilum while maintaining mobility of the wrist and simultaneously avoiding interference with the laparoscope and/or laparoscopic instruments. The non-dominant hand is placed into the operative field while the dominant hand is used to work the laparoscopic instruments.

The first step in extra-peritoneal HAL renal surgery is to gain access to the retroperitoneal space, and position a hand through an incision in the anterior abdominal wall. A 6-7 cm incision is made lateral to the rectus fascia (approximately four centimeters above the anterior superior iliac spine). The anterior abdominal wall fascia is opened lateral to the rectus abdominal sheath, through the apeuronorosis of the external oblique fascia. A space medial to the internal oblique and the transversalis muscles is developed by splitting the muscles. Next a space between the retroperitoneal and peritoneal cavities is developed. The peritoneum is swept medial and the retroperitonal space is entered. The Gelport device is used to introduce the hand 
into the retoperitoneum. The retroperitoneal cavity is insufflated with $\mathrm{CO}_{2}$. The remaining trocars are placed under direct vision (per the above described configuration).

On the left side, the White line of Toldt is incised, allowing the peritoneal cavity and the descending colon, to rotate medially. Next, the mesocolon is dissected off the anterior aspect of Gerota's fascia. The dissection is carried in a cephalad direction, freeing the splenorenal attachments. The plane between the tail of the pancreas and Gerota's fascia is developed, allowing the tail of the pancreas to fall medially. The anterior aspect of the adrenal gland and renal hilum is now exposed. Digits of the nondominant hand and instruments in the dominant hand are used to dissect the renal hilum. The gonadal and/or adrenal veins are clipped and divided.

On the right side, the peritoneum and ascending colon are mobilized medially, by incising the White line of Toldt. The liver ligaments (coronary and triangular) are divided, which exposes the upper aspect of Gerota's fascia. A liver retractor can be placed through a right upper quadrant port. Next the duodenum is kocherized, allowing the vena cava and renal hilum to be exposed.

In the subsequent step (for both the right and left side), the ureter is identified, and followed all the way to the level of the renal hilum. All of the attachments medial to the ureter are isolated and divided. After the renal vein is identified, connections to the lower pole are identified and separated. Next, lateral and posterior renal attachments are released, allowing the kidney to rotate medially, which facilitates posterior dissection of the renal vessels. At this point, the kidney is rolled anteromedially to allow dissection of the renal hilum from a posterior approach. During a radical/simple nephrectomy, the renal vessels are divided once adequate length is obtained to place a clips and staplers on the vessels. In contrast, during a donor nephrectomy, the hilar dissection is complete when the renal vein and artery are skeletanized to the inferior vena cava and aorta. The artery is divided with either an endoscopic articulating stapling device or clips. The vein is secured with an endoscopic linear stapling device. After that, the only remaining attachments to the kidney are located superiorly. If the adrenal gland is to be left in place, the upper pole attachments may be dissected from the adrenal with a ligasure device. If the adrenal gland is to be removed with the specimen, then the superior, medial, and posterior attachments of the adrenal are released. For a radical nephrectomy, the ureter and all remaining attachments can be divided and the specimen removed through the hand incision. During a donor nephrectomy, the ureter is clipped and divided below the level of the iliac vessels and the kidney is immediately handed to the recipient team for preparation.

When performing a partial nephrectomy, the entire kidney is dissected-free except for maintaining Gerota's fascia on top of the tumor. This permits access to the renal artery in case temporary occlusion is needed. A laparoscopic ultrasound is used to confirm tumor depth and map out renal vasculature. The renal capsule is circumferentially scored one half centimeter from the tumor edge. Next, laparoscopic scissors are used to excise the lesion, while the intra-abdominal hand squeezes the kidney proximal to the resection site to ensure temporary hemostasis. One of several methods may be utilized to secure hemostasis. The Ligasure device can be used to cauterize the defect. Surgicel bolsters may be placed into the defect and Vicryl pleget sutures can be used to reapproximate the renal capsule, with the aid of Lapra-tyes and Hem-0lok's. Next, hemostatic agents, such as Fibrin glue, are applied over the defect. Finally, Gerota's fascia is reapproximated to reinforce the repair. The specimen is placed in an Endocatch bag and removed via one of the port sites. A Jackson Pratt drain is secured through a lateral trocar site.

\section{Results}

From January 2010 to December 2012, eight retroperitoneal HAL nephrectomies and one retroperitoneal HAL partial nephrectomy was performed by one surgeon (NN). The average age of the patients was 56 years old, and the average body mass index was 27.5. Three patients were female and six patients were male. The indications for nephrectomy and partial nephrectomy were enhancing solid renal lesions $(n=8)$ and enhancing cystic mass $(n=1)$. All of the patients, who underwent a nephrectomy, were on dialysis prior to the kidney surgery. Two patients were on PD, and PD did not have to be interrupted postoperatively, because we did not violate the peritoneal cavity by the retroperitoneal approach. In addition, four of the nephrectomy patients had undergone previous abdominal surgery and we avoided excessive peritoneal adhesions by pursuing the retroperitoneal route. The patient who underwent a partial nephrectomy had a history of cirrhosis, and therefore the peritoneal cavity was avoided during his kidney surgery. Our results are summarized in Table 1. In the HAL group, the average operative time was $210 \mathrm{~min}$, the estimated blood loss $110 \mathrm{~mL}$,

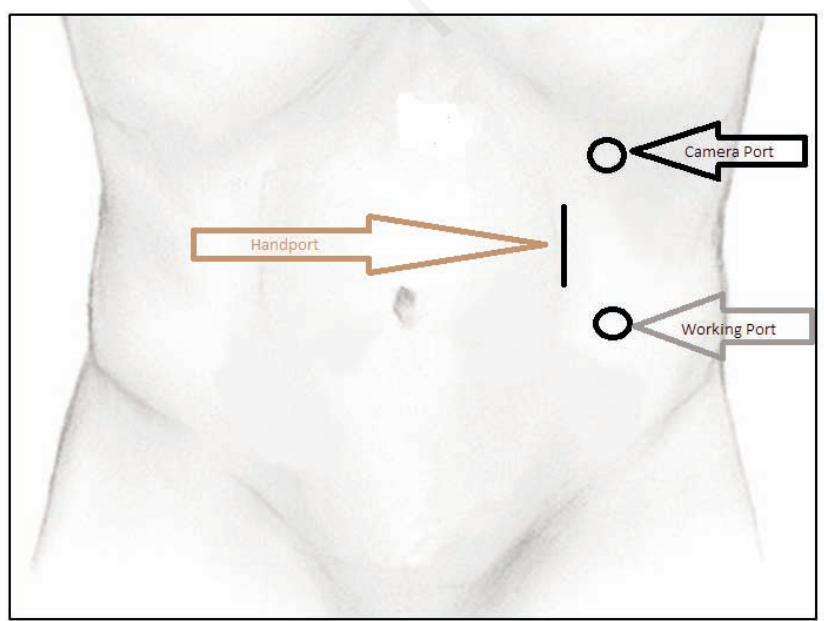

Figure 1. Port configuration for left-sided nephrectomy.

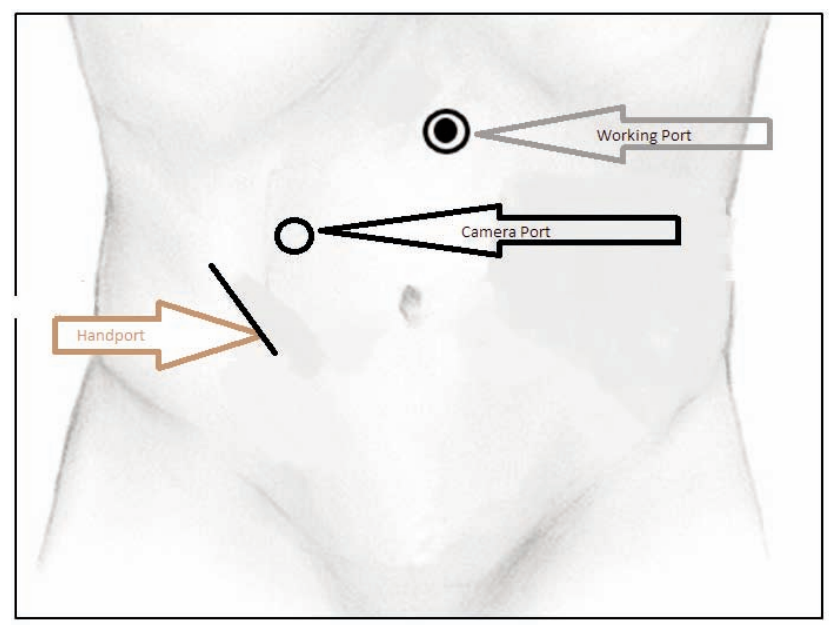

Figure 2. Port configuration for right-sided nephrectomy. 
and the average change in hematocrit was 3 units and average change in creatinine was 1 point. No patients required a blood transfusion, and none of the surgeries had to be converted to open. In the partial nephrectomy case, the renal hilum did not require occlusion. There were no intra-operative or postoperative complications in any of the cases, with a mean follow-up of fourteen months. On average, patients who underwent HAL nephrectomy, resumed oral intake in two days, and were discharged home in three days. Pathological examination revealed: two lesions were found to be benign, and seven lesions were confirmed to be malignant. Pathological evaluation found renal cell carcinoma in three tumors, papillary carcinoma in three other lesions and chromophobe renal carcinoma in one tumor. Another patient was found to have a benign renal cyst and the partial nephrectomy patient was found to have an angiomyelolipoma. Average tumor diameter was three centimeters. There were no positive surgical margins.

\section{Discussion}

Previous authors have compared outcomes of standard laparoscopic nephrectomy to hand assisted laparoscopic nephrectomy. ${ }^{2}$ With respect to parenteral and oral narcotic requirements, length of stay, and time of convalescence, Wolfe et al. ${ }^{2}$ found standard laparoscopy provided no advantage compared to HAL in a nephrectomy series. In fact, average operative times were shorter in the HAL nephrectomy series compared with the standard laparoscopy and estimated blood loss was less in the HAL donor nephrectomy series compared with the laparoscopic donor nephrectomy series. ${ }^{2}$

Similar to the mini-flank incision, handassisted laparoscopic approach offers a practical alternative to traditional open or laparo- scopic partial (PN) or radical nephrectomy (RN). ${ }^{16}$ In addition, studies show robotic assisted nephrectomy offered no advantage, and was more costly over traditional laparoscopic or hand-assisted approaches. ${ }^{17}$ Future studies will be needed to compare retroperitoneal HAL nephrectomy to open and robotic approaches.

In terms of evaluating donor nephrectomy techniques, recipient allograft function and donor outcomes are equally important. When reviewing recipient data, HAL donor nephrectomy and open donor nephrectomy had similar outcomes with nadir creatinine, time-to-nadir creatinine, and creatinine clearance. ${ }^{12-16}$ In terms of complications, the HAL group had lower incidence of ureteral obstructions and fewer episodes of rejection/ delayed graft function. ${ }^{18-22}$ In addition, authors identified distinct advantages in favor of the HAL donor nephrectomy technique over laparoscopic donor nephrectomy. HAL appears to have a decreased incidence of ureteral complications and delayed graft function compared with standard laparoscopic series. ${ }^{19,21}$ Noguiera et al. ${ }^{19}$ found a $7.6 \%$ incidence and Kavoussi ${ }^{21}$ reported a $6.4 \%$ of delayed graft function in standard laparoscopic nephrectomy series; compared to reported $1.6 \%$ in HAL donor nephrectomies series. ${ }^{20}$ In addition, ureteral complication rate of $1.6 \%$ in HAL nephrectomies, compares favorably to standard laparoscopic series that report ureteral complication rates of 4.5 and $9.1 \% .^{22}$

Retroperitoneal hand-assisted nephrectomy has been described for donor nephrectomies. Wadstrom et al. ${ }^{10}$ performed the first handassisted retroperitoneal nephrectomy on a kidney donor, in 2003. Subsequently, he compared clinical outcomes for hand assisted retroperitoneal donor nephrectomies (HARDN) $(n=14)$ to laparoscopic donor nephrectomies (LDN) $(\mathrm{n}=11) .{ }^{11}$ Operative time was found to be significantly shorter with HARDN $v s$ LDN (141 vs 270 min). ${ }^{11}$ Two additional studies, compared surgical outcomes of hand-assisted retroperitoneal donor nephrectomies (HARDN) to hand-assisted transperitoneal laparoscopic donor nephrectomies (HALDN), and found similar results (Sundqvist et al. ${ }^{12}$ - prospective study, and Gjertsen et al. ${ }^{13}$ - retrospective study). Furthermore, Dols et al. ${ }^{14}$ completed a prospective study, comparing 20 left-sided HARDN procedures and 40 left-sided LDNs. Median operation time and warm ischemia time were shorter in HARND (180 vs $225 \mathrm{~min}$, and 3 vs 5 min, respectively). ${ }^{14}$ Lastly, Leonienke et al. executed a randomized controlled trial comparing HARDN technique to LDN, with clinical end-points favoring HARDN. ${ }^{15}$

Results on HAL partial nephrectomy data were reported by Wolfe et $a l^{3}$ They compared outcomes between HAL and laparoscopic nephrectomy groups and found intraoperative and post-operative outcomes to be similar between the groups. Both methods revealed favorable outcomes with respect to convalescence data, with both groups reporting a return to normal activity within one week and an average return to work within one month. ${ }^{3}$ They also contrasted their HAL partial nephrectomy data to a contemporary series of 12 open partial nephrectomies and concluded that HAL provides an equal effective tumor removal with quicker patient recoveries. At time of follow-up (mean of 10 months), there was one distant recurrence in the open partial nephrectomy group and one local recurrence in the HAL partial nephrectomy group. ${ }^{3}$ No previous studies have described retroperitoneal HAL partial nephrectomy techniques.

We compared our retroperitoneal HAL outcomes to a contemporary group of patients that underwent nephrectomy using traditional open techniques (for solid renal masses). ${ }^{23}$ Operative time (range 45-420 min) and complication rates $(<1 \%)$ were similar between the HAL and open groups. ${ }^{23}$ However, narcotic requirements (parenteral and oral) and length

Table 1. Patient characteristics and outcomes for retroperitoneal hand-assisted laparoscopic nephrectomies.

\begin{tabular}{|c|c|c|c|c|c|c|c|c|c|c|c|}
\hline \multirow{3}{*}{$\begin{array}{l}\text { Procedure } \\
\text { Nephrectomy } \\
\text { Thtal }\end{array}$} & \multicolumn{4}{|c|}{ Patient characteristics } & \multirow[b]{2}{*}{ OR time } & \multirow[b]{2}{*}{ EBL (mL) } & \multirow[b]{2}{*}{ Het pre- } & Outcomes & \multirow[b]{2}{*}{ Cr pre- } & \multirow[b]{2}{*}{ Cr post- } & \multirow[b]{2}{*}{ LOS (days) } \\
\hline & Age & Gender & BMI & Side & & & & Het post- & & & \\
\hline & \\
\hline & 66 & M & 31.9 & $\mathrm{R}$ & $4 \mathrm{~h} 24 \mathrm{~min}$ & 100 & 31.2 & 25.9 & 6 & 8.1 & 3 \\
\hline & 42 & M & 27.5 & $\mathrm{R}$ & $3 \mathrm{~h}$ & 250 & 42.5 & 34.1 & 11.1 & 12.4 & 3 \\
\hline & 49 & $\mathrm{~F}$ & 26.6 & $\mathrm{R}$ & $2 \mathrm{~h} 25 \mathrm{~min}$ & 50 & 36.3 & 33.6 & 6.5 & 7.5 & 2 \\
\hline & 45 & M & 26.7 & $\mathrm{~L}$ & 2 h 55 min & 100 & 46.1 & 44.6 & 13.2 & 14.7 & 4 \\
\hline & 55 & $\mathrm{~F}$ & 22.8 & $\mathrm{~L}$ & $3 \mathrm{~h} 33 \mathrm{~min}$ & 50 & 32.1 & 34.6 & 7.6 & 9 & 2 \\
\hline & 65 & M & 18.8 & L & $3 \mathrm{~h} 22 \mathrm{~min}$ & 200 & 27.8 & 37.3 & 7.5 & 8.2 & 7 \\
\hline & 72 & M & 29.9 & $\mathrm{R}$ & 2 h 26 min & 100 & 27 & 27.7 & 4.6 & 4.6 & 4 \\
\hline & 56 & $\mathrm{~F}$ & 32.5 & L & $6 \mathrm{~h} 45 \mathrm{~min}$ & 100 & 40.4 & 37.4 & 0.7 & 1.2 & 5 \\
\hline Partial & 56 & M & 30.6 & $\mathrm{~L}$ & $3 \mathrm{~h} 45 \mathrm{~min}$ & 50 & 35.6 & 35.6 & 1 & 1.2 & 1 \\
\hline
\end{tabular}

BMI, body mass index; OR, operative; EBL, estimated blood loss; Hct, hematocrit; Cr, creatinine; LOS, length of stay; M. masculine; F, feminine; R, right; L, left. 
of hospital stay (average $n=3$ days) were less in the HAL group compared to the open group. ${ }^{23}$

We present a novel technique of retroperitoneal laparoscopic hand assisted nephrectomy and partial nephrectomy (for solid renal masses). Remaining purely retroperitoneal during nephrectomy has benefits in patients with intra-abdominal pathologies such as intrabdominal adhesions or those with ascites. Our data support that retroperitoneal handassisted laparoscopy provides a safe, reproducible, and minimally invasive technique to remove renal tumors. An advantage of HAL is the ability to manage larger specimens safely and reproducibly because the hand in the operative field allows for excellent retraction and maneuverability of specimens. However, extremely larger lesions occupying the retroperitoneal space may limit surgeons utilizing a retroperitoneal approach.

\section{Conclusions}

Since the introduction of the hand port in laparoscopic surgery, ten years ago, many authors have described hand assisted nephrectomies and have reviewed outcome data. ${ }^{2-6}$ None of these articles have described the hand assisted retroperitoneal laparoscopic approach (for solid renal masses). This paper presents our initial experience of eight hand-assisted retroperitoneoscopic nephrectomies and a retroperitoneal hand assisted partial nephrectomy. Retroperitoneal laparoscopic handassisted donor nephrectomy is a viable alternative for those who prefer the extraperitoneal approach for their laparoscopic procedures. Remaining completely retroperitoneal during the surgery has advantages in patients with intra-abdominal pathologies such as ascites and intrabdominal adhesions. Due to hilar anatomy, potential advantages are greatest in right (compared to left) nephrectomies. Further long-term and prospective studies are underway.

\section{References}

1. Licht MR, Novick A. Nephron sparing surgery for renal cell carcinoma. J Urolology 1993;149:1-7.

2. Wolfe JS Jr., Moon TD, Nakada SY. Handassisted laparoscopic nephrectomy: comparison to standard laparoscopic nephrectomy. J Urolology 1998;160:22-7.

3. Wolfe JS, Seifman BD, Montie JE. Nephron sparing surgery for suspected malignancy: open surgery compared to laparoscopy with selective use of hand assistance. J Urolology 2000;163:1659-64.

4. Bannenberg JJG, Meijer DW, Bannenberg $\mathrm{JH}$, et al. Hand-assisted laparoscopic nephrectomy in the pig: initial report. Minim Invasiv Ther 1996;5:483-7.

5. Nakada SY, Moon TD, Gist M, et al. Use of the pneumo sleeve as an adjunct in laparoscopic nephrectomy. Urology 1997;49:6123 .

6. Stifelman MD, Shichma S, Sosa RE. Handassisted laparoscopy. Curr Surg Tech Urol 2000;12:1-7.

7. Shalhav AL, Dunn MD, Portis AJ, et al. Laparoscopic nephroureterectomy for upper tract transitional cell cancer: the Washington University experience. J Urolology 2000;163:1100-4.

8. Stifelman MD, Sosa RE, Andrade A, et al. Hand assisted laparoscopic nephroureterectomy for the treatment of transitional cell carcinoma of the upper tract. Urology 2000;56:741-7.

9. Slakey DP, Wood JC, Hender D, et al. Laparoscopic living donor nephrectomy: Advantages of the hand-assisted method. Transplantation 1999;68:581-3.

10. Wadstrom J, Lindstrom P, Engstrom BM. Hand-assisted retroperitoneoscopic living donor nephrectomy superior to laparoscopic nephrectomy. Transplant P 2003;35: 782-3.

11. Wadstrom J. Hand-assisted retroperitoneoscopic live donor nephrectomy: experience from the first 75 consecutive cases. Transplantation 2005;80:1060-6.

12. Sundqvist P, Feuk U, Haggman M, et al. Hand-assisted retroperitoneoscopic live donor nephrectomy in comparison to open and laparoscopic procedures: a prospective study on donor morbidity and kidney function. Transplantation 2004;78:147-53.

13. Gjertsen H, Sandberg AK, Wadstrom J, et al. Introduction of hand-assisted retroperitoneoscopic living donor nephrectomy at Karolinska University Hospital Huddinge. Transplant P 2006;38:2644-5.

14. Dols LF, Kok NF, Terkivatan T, et al. Optimizing left-sided live kidney donation: hand-assisted retroperitoneoscopic as alternative to standard laparoscopic donor nephrectomy. Transplant Int 2010;23:35863.

15. Leonienke FC, Niels FM, Terkivata T. Hand-assisted retroperitoneoscopic versus standard laparoscopic donor nephrectomy: HARP-trial. BMC Surg 2010;10:1471-2482.

16. Diblasio CJ, Snyder ME, Russo P. Miniflank supra-11th rib incision for open partial or radical nephrectomy. BJU Int 2006;97:149-56.

17. Boger M, Lucas SM, Popp SC, et al. Comparison of robot-assisted nephrectomy with laparoscopic and hand-assisted laparoscopic nephrectomy. JSLS-J Soc Laparoend 2010;14:374-80.

18. Wolf JS Jr., Tchetgen MB, Merion RM. Hand-assisted laparoscopic live donor nephrectomy. Urology 1998;52:885-7.

19. Nogueira JM, Cangro CB, Fink JC, et al. A comparison of recipient renal outcomes with laparoscopic versus open live donor nephrectomy. Transplantation 1999;67: 722-8.

20. Ratner LE, Montgomery RA, Kavoussi LR. Laparoscopic live donor nephrectomy: the four year John Hopkins University experience. Nephrol Dial Transpl 1999;14:2090-3.

21. Kavoussi LR. Laparoscopic donor nephrectomy. Kidney Int 2000;57:2175-86.

22. Stifelman MD, Hull D, Sosa RE, et al. Hand assisted laparoscopic donor nephrectomy: a comparison with the open approach. J Urolology 2001;166:444-8.

23. Novick A, Derweesh I. Open partial nephrectomy for renal tumors: current status. BJU Int 2005;95(Suppl.2):35-40. 